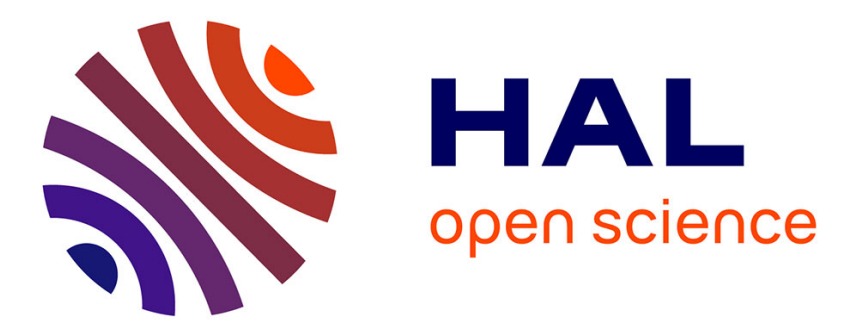

\title{
3-Bromo N -Alkyl Cyanamides as Versatile Building Blocks
}

Karen Wright, Bruno Drouillat, Laurence Menguy, Jérôme Marrot, François

Couty

\section{- To cite this version:}

Karen Wright, Bruno Drouillat, Laurence Menguy, Jérôme Marrot, François Couty. 3-Bromo N -Alkyl Cyanamides as Versatile Building Blocks. European Journal of Organic Chemistry, 2019, 2019 (1), pp.112-117. 10.1002/ejoc.201801439 . hal-02348865

\section{HAL Id: hal-02348865 https://hal.science/hal-02348865}

Submitted on 29 Jul 2021

HAL is a multi-disciplinary open access archive for the deposit and dissemination of scientific research documents, whether they are published or not. The documents may come from teaching and research institutions in France or abroad, or from public or private research centers.
L'archive ouverte pluridisciplinaire HAL, est destinée au dépôt et à la diffusion de documents scientifiques de niveau recherche, publiés ou non, émanant des établissements d'enseignement et de recherche français ou étrangers, des laboratoires publics ou privés. 
This is the peer reviewed version of the following article: Wright, K. , Drouillat, B. , Menguy, L. , Marrot, J. and Couty, F. (2019), 3-Bromo N-Alkyl Cyanamides as Versatile Building Blocks. Eur. J. Org. Chem., 2019: 112-117. doi:10.1002/ejoc.201801439, which has been published in final form at https://doi.org/10.1002/ejoc.201801439. This article may be used for non-commercial purposes in accordance with Wiley Terms and Conditions for Use of Self-Archived Versions.

\title{
3-Bromo N-Alkyl Cyanamides as Versatile Building Blocks
}

\author{
Karen Wright*, Bruno Drouillat, Laurence Menguy, Jérôme Marrot and François Couty ${ }^{\text {[a] }}$
}

\begin{abstract}
Bromo $\mathrm{N}$-alkyl cyanamides are accessible in one simple synthetic operation from $\mathrm{N}$-alkyl azetidines reacting with cyanogen bromide. These unexplored building blocks combine two extremely useful electrophilic moieties: an alkyl bromide and a cyanamide. We demonstrate here that they can be transformed into a range of nitrogen-containing molecules, and especially cyclic guanidines, accessible in enantiomerically pure form. Considering the large structural diversity of available azetidines, this contribution complements the use of these strained heterocycles in diversityoriented synthesis.
\end{abstract}

\section{Introduction}

The search for available, flexible, and bench-stable new building blocks allowing the simple generation of molecular diversity is a key topic in the context of diversity-oriented synthesis (DOS), which has recently emerged as a useful tool for the discovery of new small bioactive molecules. ${ }^{1}$ Azetidines, as reactive yet available $^{2}$ and bench-stable nitrogen heterocycles, have emerged as promising candidates to generate new libraries with high levels of structural diversity, ${ }^{3}$ from which new drug candidates have emerged. ${ }^{4}$ We recently reported ${ }^{5}$ that $\mathrm{N}$-alkyl azetidines 1 efficiently react with cyanogen bromide, leading to regioisomeric 3-bromo $\mathrm{N}$-alkyl cyanamides 2-3 through the von Braun reaction. ${ }^{6}$ These compounds are produced with predictable and usually high regioselectivity in the ring-opening step, and they can be decorated with diverse substituents or functional groups starting from the required azetidines, which are very easy to prepare (Scheme 1 ).<smiles>[R]C1C([R])N([R1])C1[R]</smiles>

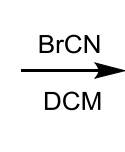<smiles>[R]C(Br)C([R])N([R])N</smiles><smiles>[R]C(Br)C([R])N([R])N</smiles>

Scheme 1. Reaction of azetidines with cyanogen bromide (von Braun reaction).

These 3-bromo $\mathrm{N}$-alkyl cyanamides combine two useful electrophilic moieties: the cyanamide ${ }^{7}$ and an alkyl bromide.

[a] Institut Lavoisier de Versailles, UMR 8180

Université de Versailles St-Quentin-en-Yvelines, Université Paris Saclay.

45 av. des Etats-Unis, 78035 Versailles Cedex, France

\section{E-mail: karen.wright@uvsq.fr; http://www.ilv.uvsq.fr Fax: $+33(0) 139254452$}

Supporting information for this article is given via a link at the end of the document.

They can be easily fitted with additional functional groups such as a nitrile $\left(R^{2}\right.$ or $\left.R^{4}\right)$, and can be produced in enantiomerically pure form, starting from an azetidine derived from a $\beta$-aminoalcohol. ${ }^{2 f, g}$ This article aims to explore the reactivity of these original building blocks, in order to generate structural diversity.

\section{Results and Discussion}

We first focused on the synthesis of 6-membered cyclic guanidines through the reaction of a 3-bromo $\mathrm{N}$-cyanamide 4, easily prepared from commercially available $N$-benzhydryl azetidine, with primary amines ${ }^{5}$ (Scheme 2, Table 1). Such reactions were first described by Hageman $^{8}$ in the early fifties and by $\mathrm{us}^{5}$ in a single example, but its scope remained to be established. Interest in guanidines has recently been growing, especially in the field of organocatalysis, ${ }^{9}$ and the combination of these simple reactions, together with the potential structural diversity brought by the two reaction partners, would represent a convenient entry to these heterocycles.

Our initial attempts at guanidine synthesis, based on Hageman's conditions (refluxing ethanol), needed however protracted reaction times to reach completion, and gave low yields in the case of hindered amines. An optimisation study was therefore carried out with the sterically hindered 1-adamantylamine and cyclohexylamine, and it was found that a spectacular shortening of reaction time was possible upon microwave irradiation. Optimal conditions were found with isopropanol as solvent at $120^{\circ} \mathrm{C}$ (Table 1). Solvent effects were less obvious, and the use of HFIP, reported to be a solvent promoting the addition of<smiles>[R]N1CCCN(C(=N)c2ccccc2)C1CCCCC</smiles>

Scheme 2. Synthesis of cyclic guanidines by reaction of 3-bromo $\mathrm{N}$-alkyl cyanamide $\mathbf{4}$ with amines.

amines to cyanamides ${ }^{10}$ was not beneficial here. From a practical viewpoint, this reaction directly affords guanidinium hydrobromides conveniently isolated as crystalline salts. 


\begin{tabular}{|c|c|c|c|c|}
\hline Amine & Conditions & Time & Yield & Product \\
\hline $\mathrm{R}=\mathrm{Cy}$ & $80^{\circ} \mathrm{C}, \mathrm{EtOH}$ & 10 days & $84 \%$ & $5, R=C y$ \\
\hline $\mathrm{R}=\mathrm{Cy}$ & $\begin{array}{l}110^{\circ} \mathrm{C}, \\
\text { Toluene }\end{array}$ & 4 days & $71 \%$ & $5, R=C y$ \\
\hline $\mathrm{R}=\mathrm{Cy}$ & $\begin{array}{l}110^{\circ} \mathrm{C} \\
\mathrm{BuOH}\end{array}$ & 4 days & $82 \%$ & 5, $R=C y$ \\
\hline$R=C y$ & $\begin{array}{l}\mu \mathrm{w}, 80^{\circ} \mathrm{C} \\
\mathrm{EtOH}\end{array}$ & $4 \mathrm{~h}$ & $73 \%$ & 5, $R=C y$ \\
\hline $\mathrm{R}=\mathrm{Cy}$ & $\begin{array}{l}\mu \mathrm{w}, 80^{\circ} \mathrm{C} \\
\mathrm{PrOH}\end{array}$ & $4 \mathrm{~h}$ & $85 \%$ & 5, $R=C y$ \\
\hline $\mathrm{R}=\mathrm{Cy}$ & $\begin{array}{l}\mu \mathrm{w}, 120^{\circ} \mathrm{C} \\
\mathrm{PrOH}\end{array}$ & $1 \mathrm{~h}$ & $82 \%$ & 5, R = Cy \\
\hline
\end{tabular}

$\mathrm{R}=$ Adamantyl $\quad 80^{\circ} \mathrm{C}, \mathrm{EtOH} \quad 10$ days $\quad \begin{array}{lll}16 \% & \mathbf{6}, \quad \mathrm{R} \\ & & \text { Adamantyl }\end{array}=$

$\begin{array}{llllll}\mathrm{R}=\text { Adamantyl } & \begin{array}{l}110^{\circ} \mathrm{C}, \\ \mathrm{BuOH}\end{array} & n- & 4 \text { days } & 71 \% & \mathbf{6}, \quad \mathrm{R} \\ & & & \text { Adamantyl }\end{array}=$

$\begin{array}{lllll}\mathrm{R}=\text { Adamantyl } & \begin{array}{l}\mu \mathrm{w}, 120^{\circ} \mathrm{C} \\ i-\mathrm{PrOH}\end{array} & 3 \mathrm{~h} & 75 \% & \mathbf{6}, \mathrm{R} \\ & & & \text { Adamantyl }\end{array}=$

Table 1: Optimization of reaction conditions for hindered amines.

The reaction scope was next examined with other amines in either refluxing ethanol $\left(80^{\circ} \mathrm{C}, 7\right.$ days: conditions $\left.A\right)$ or under 1 :

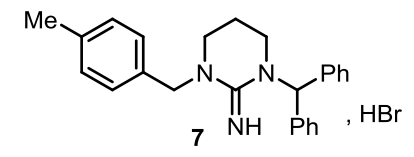<smiles>COc1ccc(N2CCCN(C(=N)C(Br)c3ccccc3)C2c2ccccc2)cc1</smiles><smiles>CC(C)(C)OC(=O)NCCN1CCCN(C(=N)c2ccccc2)C1c1ccccc1</smiles>

Conditions A: $78 \%$ Conditions B: $78 \%$<smiles>N=C1N(CCCN2CCCN(C(c3ccccc3)c3ccccc3)C2=N)CCCN1C(Br)c1ccccc1</smiles>

Conditions A: $87 \%$
Conditions A: $72 \%$

8 $T_{\mathrm{Nh}}, \mathrm{HBr}$

Conditions A: $67 \%$ Conditions B: $91 \%$<smiles>C=CCN1CCCN(C(c2ccccc2)c2ccccc2)C1=N</smiles>

10

Conditions B: $87 \%$

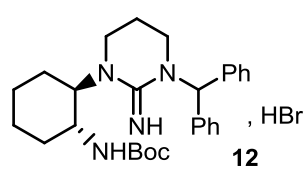

Conditions A: $88 \%$<smiles>N=C1N(C(c2ccccc2)c2ccccc2)CCCN1[C@@H]1CCCCN1C(=N)N1CCCN(c2ccccc2)C1c1ccccc1</smiles>

Amine scope. a) Stoichiometry of $1: 2$ for $(1 R, 2 R)$-cyclohexyl-1,2-diamine : 4 was used.

$\mu$ wave irradiation in $i-\mathrm{PrOH}\left(120^{\circ} \mathrm{C}, 1-3 \mathrm{~h}, 200 \mathrm{~W}\right.$ : conditions $\left.B\right)$. The structures of the produced guanidines 7-14 are shown in Figure 1, demonstrating a wide scope for this reaction, including for aliphatic amines and anilines, as well as for functionalized amines and diamines. In this last case, the crystalline bis- Figure guanidines $\mathbf{1 3}$ and $\mathbf{1 4}$ were isolated in average to good yields, thus demonstrating that the initial alkylation step is probably more rapid than the cyclisation step leading to the 6-membered guanidine. ${ }^{11}$ The structure of the $\mathrm{C} 2$-symmetric chiral guanidine 14 , obtained from enantiomerically pure $(1 R, 2 R)$-cyclohexyl-1,2diamine was determined by X-ray crystallography. ${ }^{12}$ It should be mentioned here that beta amino alcohols, amino esters, or other 1,2-diamines such as $(R, R)$-1,2-diphenylethane-1,2-diamine failed to produce the expected guanidines under conditions $A$ or B. Only (S)-phenylglycine derived amino ester $\mathbf{1 5}$ gave bicyclic guanidine 16 in low isolated yield. This compound displayed no optical activity, probably due to complete epimerization. Noteworthy also is the formation of the 7 -membered $[1,2,4]$ triazepane 17 when phenylhydrazine was used under conditions $B$. The structure of this rarely reported heterocyclic scaffold ${ }^{13}$ was determined by X-ray crystallography, and its NMR spectra display two distinct conformers. ${ }^{12}$ The position of the $\mathrm{N}-\mathrm{Ph}$ moiety in 17 can be explained by the known propensity of hydrazines to undergo alkylation at their more substituted nitrogen atom. ${ }^{14} \mathrm{~N}$-phenyl- $\mathrm{N}$-methylhydrazine only gave a complex mixture of products under conditions B (Scheme 3 ). 
Finally, the scope of the reacting 3-bromocyanamide was briefly examined. Thus the 3-bromo cyanamides substituted at C-1 18 and $19{ }^{5}$ gave good yields of substituted guanidines 21 and 22 . Notably, no competing reactivity of the cyano group in 19 was observed, but $t$-butyl ester $\mathbf{2 0}$ behaved differently, since only low yields of the expected guanidine $\mathbf{2 3}$ could be isolated under conditions $A$. The major isolated product resulted in this case from an intramolecular cyclization involving the $t$-butyl ester acting as the nucleophile, yielding lactone $\mathbf{2 4}$ in 58\% yield upon $\mu$ wave irradiation in $\mathrm{EtOH}$ at $80^{\circ} \mathrm{C}$. This lactone could also be obtained by simply heating in $\mathrm{HFIP}\left(50^{\circ} \mathrm{C}, 7\right.$ days $)$ without added amine. Finally, the 1,2-disubstituted 3-bromo cyanamide 25 was evaluated under conditions $B$ with 4-methylbenzylamine, but the expected guanidine was not produced. Instead, cyclopropane 26

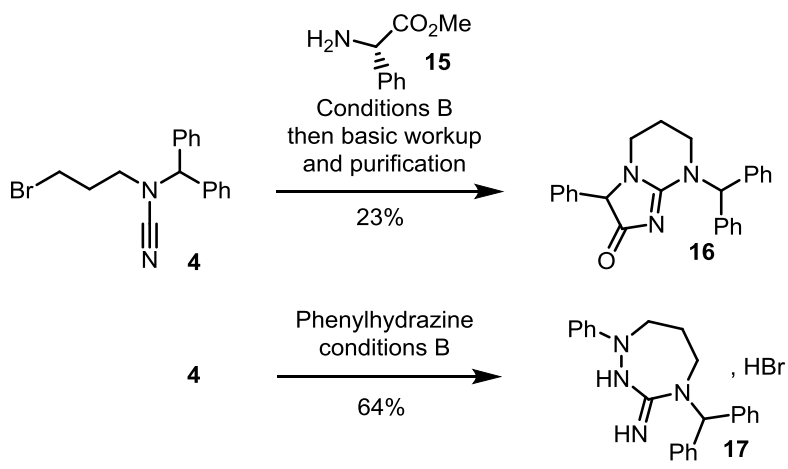

Scheme 3. Reaction with other functionalized amines and hydrazines.

was isolated from the reaction mixture, in which the relative stereochemistry of the substituents was determined by X-ray crystallography. ${ }^{12}$ This demonstrates that C-2 substitution in the starting cyanamide is detrimental to guanidine formation, probably by lowering the electrophilic character of the alkyl bromide. Thus, the amine acts in this case as a base, able to deprotonate the position alpha to the nitrile, leading to intramolecular alkylation. A further experiment was performed to confirm this, by treatment of $\mathbf{2 5}$ with LiHMDS (a non-nucleophilic base) in THF at $-78^{\circ} \mathrm{C}$. In this case, the cyclopropanes 26 and 27 were isolated in 55 and $31 \%$ yield respectively (Scheme 4).

\section{Conclusions}

This work demonstrates that 3-bromo- $N$-alkyl cyanamides are versatile building blocks able to generate in a single step various heterocyclic structures. Such building blocks are very easy to prepare from azetidines, for which synthetic access has widened only recently, allowing the introduction of a vast array of substituents and solving the issues of diastereoselectivity and enantioselectivity. ${ }^{2}$ The production of libraries of 3-bromo- $\mathrm{N}$-alkyl-cyanamides may therefore.benefit from the progress made in azetidine chemistry, and appear, as demonstrated here, as innovative tools for DOS strategies.<smiles>C[C@H](N)[C@H](OCc1ccccc1)c1ccccc1</smiles>

(1: 1 diastereoisomeric mixture)<smiles>Cc1ccc(CN)cc1CN1CCC(C#N)N(Cc2ccccc2)C1=N</smiles>

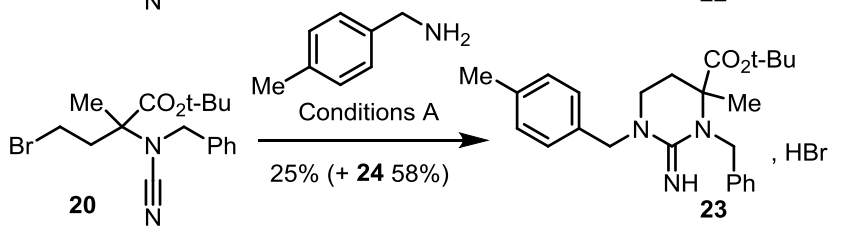

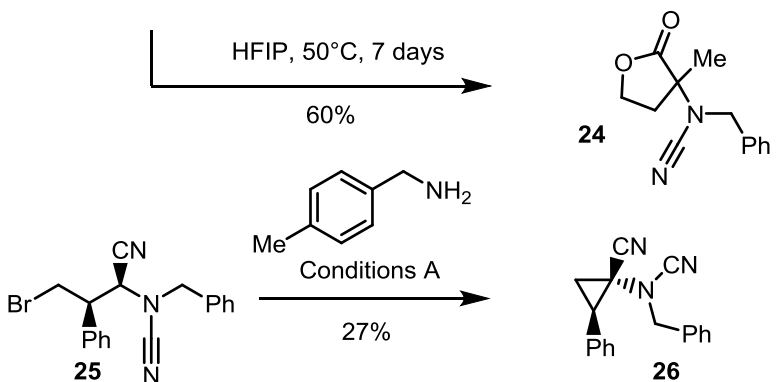

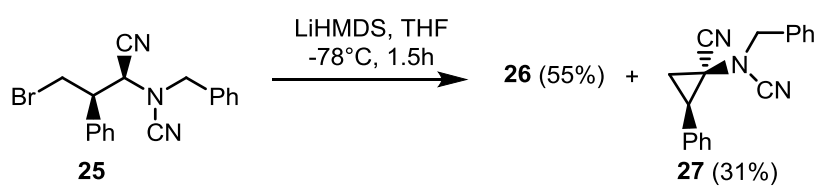

Scope of the 3-bromo-cyanamide reactant

\section{Experimental Section}

General information: ${ }^{1} \mathrm{H}$ and ${ }^{13} \mathrm{C}$ NMR spectra were recorded at 300 and $75 \mathrm{MHz}$, respectively; chemical shifts ( $\delta$ ) are reported in ppm and coupling constants $(\mathcal{J})$ reported in Hertz and rounded up to $0.1 \mathrm{~Hz}$. Splitting patterns are abbreviated as follows: singlet (s), doublet (d) Scheme 4. triplet ( $\mathrm{t})$, quartet (q), quintuplet (quint.), multiplet $(\mathrm{m})$, broad (br), or a combination of these. Solvents were used as internal standard when assigning NMR spectra $\left(\delta \mathrm{H}: \mathrm{CDCl}_{3} 7.27 \mathrm{ppm} ; \delta \mathrm{C}: \mathrm{CDCl}_{3} 77.0\right.$ ppm; $\left.\delta \mathrm{H}: \mathrm{CD}_{3} \mathrm{OD} 3.34 \mathrm{ppm}, \delta \mathrm{C}: \mathrm{CD}_{3} \mathrm{OD} 49.86 \mathrm{ppm}\right)$. Assignments for signals from ${ }^{1} \mathrm{H}$ and ${ }^{13} \mathrm{C}$ in the NMR spectra were validated by twodimensional correlated spectroscopy (2D COSY and HSQC) and Heteronuclear Multiple Bond Correlation (HMBC). High-resolution mass spectra (HR-MS) were obtained on a Q-TOF mass spectrometer. IR data were collected with an ATR-FT-IR spectrometer. THF was distilled from sodium/benzophenone ketyl. Column chromatography was performed on 
silica gel (230-400 mesh) with use of various mixtures of EtOAc, petroleum ether $\left(35-60^{\circ} \mathrm{C}\right.$ fraction) (PE) and methanol. TLC was performed on Merck Kieselgel 60 F254 plates. Melting points are uncorrected. All reactions under microwave irradiation were performed in a $10 \mathrm{~mL}$ sealed tube in a commercially available monomode reactor (CEM Discover) with IR monitoring and non-invasive pressure transducer.

General procedure for the synthesis of guanidines: A solution of the required $\mathrm{N}$-(3-bromopropyl)cyanamide (1 or 2 equiv.) and of the required amine ( 1 equiv., concentration: $0.2 \mathrm{M}$ ) was heated either at $80^{\circ} \mathrm{C}$ in abs. $\mathrm{EtOH}$ (3-5 mL) under argon for 3-7 days (conditions $A$ ) or under microwave irradiation $(200 \mathrm{~W})$ at $120^{\circ} \mathrm{C}$ in $\mathrm{iPrOH}(4-5 \mathrm{~mL})$ for $1-3$ hours (conditions $B$ ). The progress of the reaction was monitored by TLC analysis. The solution was concentrated under reduced pressure, and the residue was triturated with small portions of diethyl ether. The resulting solid was washed with EtOAc and dried under reduced pressure.

\section{1-Benzhydryl-3-cyclohexyl-3,4,5,6-tetrahydropyrimidin-2(1 H)- ylideneamine hydrobromide 5}

White solid; $\mathrm{Mp}$ : $225-228^{\circ} \mathrm{C}$; $\mathrm{R}_{\mathrm{f}}$ : 0.23 (DCM/MeOH/aq. $30 \% \quad \mathrm{NH}_{4} \mathrm{OH}$ 95/5/1): ${ }^{1} \mathrm{H}$ NMR $\left(300 \mathrm{MHz}, \mathrm{CD}_{3} \mathrm{OD}\right): \delta=7.47-7.37(\mathrm{~m}, 6 \mathrm{H}, \mathrm{ArH}), 7.25-$ $7.22(\mathrm{~m}, 4 \mathrm{H}, \mathrm{ArH}), 6.56(\mathrm{~s}, 1 \mathrm{H}, \mathrm{NCHPh}), 3.89-3.79(\mathrm{~m}, 1 \mathrm{H}, \mathrm{CHN}), 3.36(\mathrm{t}$ $\left.J=5.9 \mathrm{~Hz}, 2 \mathrm{H}, \mathrm{CH}_{2} \mathrm{~N}\right), 3.10\left(\mathrm{t}, \mathrm{J}=5.8 \mathrm{~Hz}, 2 \mathrm{H}, \mathrm{CH}_{2} \mathrm{~N}\right), 1.91-1.83(\mathrm{~m}, 6 \mathrm{H}$, $\left.\mathrm{CH}_{2}, \mathrm{CH}_{2 \mathrm{cyc}}\right), 1.71-1.63$ (m, 3H, $\left.\mathrm{CH}_{2 \mathrm{cyc}}\right), 1.54-1.42\left(\mathrm{~m}, 2 \mathrm{H}, \mathrm{CH}_{2 \mathrm{cyc}}\right), 1.26-$ $1.13\left(\mathrm{~m}, 1 \mathrm{H}, \mathrm{CH}_{2 \mathrm{cyc}}\right) \mathrm{ppm} .{ }^{13} \mathrm{C}$ NMR $\left(75 \mathrm{MHz}, \mathrm{CD}_{3} \mathrm{OD}\right): \delta=155.4(\mathrm{C}=\mathrm{NH})$ $138.6\left(\mathrm{C}_{\mathrm{qAr}}\right), 130.2,130.0,129.7\left(\mathrm{CH}_{\mathrm{Ar}}\right), 67.0\left(\mathrm{CHPh}_{2}\right), 59.6(\mathrm{CHN}), 44.6$, $41.3\left(\mathrm{CH}_{2} \mathrm{~N}\right), 30.9,26.5,26.3,22.9\left(\mathrm{CH}_{2}\right)$ ppm. IR: vmax $=3282(\mathrm{~b})$, 3108(b), 2939, 2860, 1637, 1558, 1530, 1444, 1313, 1151, 1073, 1025, $774,726,698 \mathrm{~cm}^{-1}$. HRMS (TOF MSES positive mode) $\mathrm{m} / z$ calcd. for $\mathrm{C}_{23} \mathrm{H}_{30} \mathrm{~N}_{3}[\mathrm{M}]^{+}:$: 348.2440; found : 348.2431 .

\section{1-Adamantan-1-yl-3-benzhydryl-3,4,5,6-tetrahydropyrimidin-2(1 H)-} ylideneamine hydrobromide 6

White solid; $\mathrm{Mp}$ : $209-211^{\circ} \mathrm{C}$; $\mathrm{R}_{\mathrm{f}}$ : 0.45 (DCM/MeOH/aq. $30 \% \mathrm{NH}_{4} \mathrm{OH}$ 90/10/1): ${ }^{1} \mathrm{H}$ NMR $\left(300 \mathrm{MHz}, \mathrm{CD}_{3} \mathrm{OD}\right):$ ): $\delta=7.51-7.41$ (m, $\left.6 \mathrm{H}, \mathrm{Ar}\right), 7.30$ 7.28 (m, 4H, Ar), 6.57 (s, $1 \mathrm{H}, \mathrm{NCHPh}_{2}$ ), 3.41 (appt t, J=5.9 Hz, 2H, $\mathrm{CH}_{2} \mathrm{~N}$ ), 3.27 (appt t, J=5.8 Hz, 2H, CH $\mathrm{CH}_{2} \mathrm{~N}$ ), 2.27-2.15 (m, 7H, $\mathrm{CH}_{2}, \mathrm{CHcyc}$, $\left.\mathrm{CH}_{2} \mathrm{Cyc}\right), 1.95-1.91$ (m, 2H, $\left.\mathrm{CH}_{2} \mathrm{Cyc}\right), 1.86-1.72$ (m, 8H, $\left.\mathrm{CH}_{2} \mathrm{cyc}\right)$ ppm. ${ }^{13} \mathrm{C}$ NMR $\left(75 \mathrm{MHz}, \mathrm{CD}_{3} \mathrm{OD}\right): \delta=159.2(\mathrm{C}=\mathrm{NH}), 139.8,139.1\left(\mathrm{C}_{\mathrm{qAr}}\right), 130.4$, 130.3, 129.9, 129.8, $129.6\left(\mathrm{CH}_{\mathrm{Ar}}\right), 67.0\left(\mathrm{NCHPh}_{2}\right), 62.3\left(\mathrm{C}_{\mathrm{qcyc}} \mathrm{N}\right), 43.3$ $\left(\mathrm{CH}_{2} \mathrm{~N}\right), 41.8\left(\mathrm{CH}_{2} \mathrm{~N}\right), 41.7,41.6,39.6,36.8,36.7,36.6\left(\mathrm{CH}_{2}\right), 31.7(\mathrm{CH})$, $30.7(\mathrm{CH}), 30.6(\mathrm{CH}), 28.4\left(\mathrm{CH}_{2}\right)$ ppm. IR: vmax $=3270,3061,2895$, 2848, 2205, 1633, 1526, 1495, 1451, 1361, 1065, 730, $694 \mathrm{~cm}^{-1}$. HRMS (TOF MSES positive mode) $\mathrm{m} / z$ calcd. for $\mathrm{C}_{27} \mathrm{H}_{34} \mathrm{~N}_{3}[\mathrm{M}]^{+}:$: 400.2753; found : 400.2750 .

\section{1-Benzhydryl-3-(4-methylbenzyl)-3,4,5,6-tetrahydropyrimidin-2(1 H)-} ylideneamine hydrobromide 7

White solid (72\%, conditions A); Mp: $228^{\circ} \mathrm{C}$; Rf: 0.25 (EtOAc/MeOH $/ 40 \%$ aq. $\left.\mathrm{NH}_{3}: 94 / 5 / 1\right) ;{ }^{1} \mathrm{H}$ NMR $\left(300 \mathrm{MHz}, \mathrm{CD}_{3} \mathrm{OD}\right): \delta=7.51-7.20(\mathrm{~m}, 14 \mathrm{H}$, $\mathrm{ArH}), 6.63(\mathrm{~s}, 1 \mathrm{H}, \mathrm{NCHPh} 2), 4.72\left(\mathrm{~s}, 2 \mathrm{H}, \mathrm{NCH}_{2} \mathrm{Ph}\right), 3.41$ (appt t, $2 \mathrm{H}$, $J=6.0 \mathrm{~Hz}, \mathrm{CH}_{2} \mathrm{~N}$ ), 3.20 (appt t, $2 \mathrm{H}, J=6.0 \mathrm{~Hz}, \mathrm{CH}_{2} \mathrm{~N}$ ), $2.37\left(\mathrm{~s}, 3 \mathrm{H}, \mathrm{CH}_{3}\right.$ ), 1.91 (appt quint., $2 \mathrm{H}, \mathrm{J}=6.0 \mathrm{~Hz}, \mathrm{CH}_{2}$ ) ppm. ${ }^{13} \mathrm{C} \mathrm{NMR}\left(75 \mathrm{MHz}, \mathrm{CD}_{3} \mathrm{OD}\right): \delta$ $=156.0(\mathrm{C}=\mathrm{NH}), 139.2,138.3\left(\mathrm{C}_{\mathrm{gAr}}\right), 133.0,130.8,130.1,129.9,129.7$, 129.4, $128.2\left(\mathrm{CH}_{\mathrm{Ar}}\right), 66.8(\mathrm{CH}), 54.9,47.7,44.4,22.4\left(\mathrm{CH}_{2}\right), 21.2\left(\mathrm{CH}_{3}\right)$ ppm. IR: vmax $=3346,3269,3124,2918,2202,1643,1590,1574,1545$, $1451,1328,1180,1075,1030,789,722,694 \mathrm{~cm}-1$. HRMS (TOF MSES positive mode) $\mathrm{m} / \mathrm{z}$ calcd for $\mathrm{C}_{25} \mathrm{H}_{28} \mathrm{~N}_{3}[\mathrm{M}]^{+}: 370.2283$; found : 370.2273 .

\section{(S)-1-Benzhydryl-3-(1-phenylethyl)-3,4,5,6-tetrahydropyrimidin-} 2(1H)-ylideneamine hydrobromide 8

White powder $\left(67 \%\right.$, conditions A); Mp: $162^{\circ} \mathrm{C}$; Rf: 0.25 (EtOAc/MeOH/40\% aq. $\left.\mathrm{NH}_{3}: 94 / 5 / 1\right) ;{ }^{1} \mathrm{H}$ NMR $\left(300 \mathrm{MHz}, \mathrm{CD}_{3} \mathrm{OD}\right.$ ): $\delta=$ 7.52-7.28 (m, 15H, ArH), 6.65 (s, 1H, NCHPh $), 5.47$ (q, $1 \mathrm{H}, J=6.9 \mathrm{~Hz}$,
$\mathrm{CHCH}_{3} \mathrm{Ph}$ ), 3.32-3.25 (m, $\left.1 \mathrm{H}, \mathrm{CH} / \mathrm{N}\right), 3.11$ (appt t, $2 \mathrm{H}, \mathrm{J}=6.6 \mathrm{~Hz}, \mathrm{CH}_{2} \mathrm{~N}$ ), 3.03-2.95 (m, $1 \mathrm{H}, \mathrm{CH} H \mathrm{~N}), 1.92-1.68\left(\mathrm{~m}, 2 \mathrm{H}, \mathrm{CH}_{2}\right), 1.73(\mathrm{~d}, 3 \mathrm{H}, \mathrm{J}=6.9 \mathrm{~Hz}$, $\left.\mathrm{CH}_{3}\right) \mathrm{ppm} .{ }^{13} \mathrm{C}$ NMR $\left(75 \mathrm{MHz}, \mathrm{CD}_{3} \mathrm{OD}\right): \delta=156.5(\mathrm{C}=\mathrm{NH}), 140.3,139.2$, $139.1\left(\mathrm{C}_{\mathrm{qAr}}\right), 131.0,130.5,130.4,130.3,129.1\left(\mathrm{CH}_{\mathrm{Ar}}\right), 67.7\left(\mathrm{NCHPh}_{2}\right)$ $58.6\left(\mathrm{NCHCH}_{3}\right), 45.8,42.4\left(\mathrm{NCH}_{2}\right), 23.3\left(\mathrm{CH}_{2}\right), 17.6\left(\mathrm{CH}_{3}\right)$ ppm. IR: vmax $=3301,3120,3057,3025,2974,2935,2872,1637,1557,1529$, 1444, 1380, 1321, 1069, 1026, 753, $695 \mathrm{~cm}^{-1}$. HRMS (TOF MSES positive mode) $\mathrm{m} / z$ calcd for $\mathrm{C}_{25} \mathrm{H}_{28} \mathrm{~N}_{3}[\mathrm{M}]^{+}: 370.2283$; found : 370.2275 $\left[\alpha{ }_{578}^{20}:-44(c 1.8, \mathrm{MeOH})\right.$.

\section{1-Benzhydryl-3-(4-methoxyphenyl)-3,4,5,6-tetrahydropyrimidin-} $2(1 \mathrm{H})$-ylideneamine hydrobromide 9

Purple brown solid $\left(61 \%\right.$, conditions A); $\mathrm{Mp}: 135^{\circ} \mathrm{C}$; Rf: 0.30 (EtOAc/MeOH/40\% aq. $\left.\mathrm{NH}_{3}: 93 / 5 / 2\right) ;{ }^{1} \mathrm{H}$ NMR $\left(300 \mathrm{MHz}, \mathrm{CD}_{3} \mathrm{OD}\right): \delta=$ 7.53-7.33 (m, 12H, ArH), 7.13-7.08 (m, 2H, ArH), $6.62\left(\mathrm{~s}, 1 \mathrm{H}, \mathrm{NCHPh}_{2}\right)$ $3.86\left(\mathrm{~s}, 3 \mathrm{H}, \mathrm{CH}_{3} \mathrm{OPh}\right.$ ), 3.69 (appt t, $2 \mathrm{H}, \mathrm{J}=6.0 \mathrm{~Hz}, \mathrm{CH}_{2} \mathrm{~N}$ ), 3.34-3.31 (m, $2 \mathrm{H}, \mathrm{CH}_{2} \mathrm{~N}$ ), 2.15 (appt quint., $2 \mathrm{H}, \mathrm{J}=6.0 \mathrm{~Hz}, \mathrm{CH}_{2}$ ) ppm. ${ }^{13} \mathrm{C}$ NMR $(75 \mathrm{MHz}$, $\left.\mathrm{CD}_{3} \mathrm{OD}\right): \delta=162.5(\mathrm{C}=\mathrm{NH}), 156.7,139.0,135.4\left(\mathrm{C}_{\mathrm{gAr}}\right), 130.9,130.7$, 130.5, $117.7\left(\mathrm{CH}_{\mathrm{Ar}}\right), 67.5\left(\mathrm{NCHPh}_{2}\right), 57.0\left(\mathrm{OCH}_{3}\right), 52.5,45.4\left(\mathrm{NCH}_{2}\right)$, $23.3\left(\mathrm{CH}_{2}\right)$ ppm. IR: vmax $=3444,3361,3258,3180,3132,1622,1590$ $1503,1448,1322,1239,1164,1030,837,730,698 \mathrm{~cm}^{-1}$. HRMS (TOF MSES positive mode) $\mathrm{m} / \mathrm{z}$ calcd for $\mathrm{C}_{24} \mathrm{H}_{26} \mathrm{~N}_{3} \mathrm{O}[\mathrm{M}]^{+}: 372.2076$; found 372.2077 .

\section{1-Allyl-3-benzhydryl-3,4,5,6-tetrahydropyrimidin-2(1H)-ylideneamine} hydrobromide 10

Light brown solid (88\%, conditions B); Mp: $146^{\circ} \mathrm{C}$; Rf: 0.25 (EtOAc/MeOH/40\% aq. $\left.\mathrm{NH}_{3}: 93 / 5 / 2\right) ;{ }^{1} \mathrm{H}$ NMR $\left(300 \mathrm{MHz}, \mathrm{CD}_{3} \mathrm{OD}\right.$ ): $\delta=$ 7.17-7.06 (m, 6H, ArH), 6.96-6.94 (m, 4H, ArH), $6.28(\mathrm{~s}, 1 \mathrm{H}, \mathrm{NCHPh})$, 5.66-5.53 (ddt, $\left.1 \mathrm{H}, 10.5,17.4 \mathrm{~Hz}, J=6.0, \mathrm{CH}_{2} \mathrm{CH}=\mathrm{CH}_{2}\right), 5.03-5.00(\mathrm{bd}, 1 \mathrm{H}$, $\left.J=9.9 \mathrm{~Hz}, \mathrm{CH}_{2} \mathrm{CH}=\mathrm{CHH}\right), 5.00-4.95\left(\mathrm{bd}, 1 \mathrm{H}, \mathrm{J}=16.8 \mathrm{~Hz}, \mathrm{CH}_{2} \mathrm{CH}=\mathrm{CH} H\right.$ 3.82 (appt d, $2 \mathrm{H}, J=5.1 \mathrm{~Hz}, \mathrm{CH}_{2} \mathrm{CH}=\mathrm{CH}_{2}$ ), 3.13 (appt t, $2 \mathrm{H}, J=6.1 \mathrm{~Hz}$, $\mathrm{NCH}_{2}$ ), 2.88 (appt t, $2 \mathrm{H}, \mathrm{J}=5.7 \mathrm{~Hz}, \mathrm{NCH}_{2}$ ), 1.61 (appt quint., $2 \mathrm{H}, \mathrm{J}=5.9 \mathrm{~Hz}$, $\left.\mathrm{CH}_{2}\right)$ ppm. ${ }^{13} \mathrm{C} \mathrm{NMR}\left(75 \mathrm{MHz}, \mathrm{CD}_{3} \mathrm{OD}\right): \delta=156.4(\mathrm{C}=\mathrm{NH}), 139.2\left(\mathrm{C}_{\mathrm{aAr}}\right)$, 132.7, 130.9, $130.6\left(\mathrm{CH}_{\mathrm{Ar}}\right), 130.4\left(\mathrm{CH}=\mathrm{CH}_{2}\right), 118.8\left(\mathrm{CH}=\mathrm{CH}_{2}\right), 67.4$ $\left(\mathrm{NCHPh}_{2}\right), 55.1,48.6,45.1\left(\mathrm{NCH}_{2}\right), 23.1\left(\mathrm{CH}_{2}\right) \mathrm{ppm} . \mathrm{IR}: \mathrm{vmax}=3282$, $3132,3077,3026,2959,2205,1633,1574,1539,1444,1330,1073,935$, $750,695 \mathrm{~cm}^{-1}$. HRMS (TOF MSES positive mode) $\mathrm{m} / z$ calcd for $\mathrm{C}_{20} \mathrm{H}_{24} \mathrm{~N}_{3}$ $[\mathrm{M}]^{+}:$306.1970; found : 306.1971.

\section{[2-(3-Benzhydryl-3,4,5,6-tetrahydropyrimidin-2(1H)-ylidenamino)-} ethyl] carbamic acid tert-butyl ester hydrobromide 11

Beige solid (78\%, conditions A); $\mathrm{Mp}$ : $203-204^{\circ} \mathrm{C}$; $\mathrm{R}_{\mathrm{f}}$ : 0.20 (DCM/MeOH/aq. 30\% NH $\left.\mathrm{NH}_{4} \mathrm{OH} 90 / 10 / 1\right) ;{ }^{1} \mathrm{H}$ NMR $\left(300 \mathrm{MHz}, \mathrm{CD}_{3} \mathrm{OD}\right): \delta=$ 7.46-7.40 (m, 6H, ArH), 7.27-7.25 (m, 4H, ArH), $6.55(\mathrm{~s}, 1 \mathrm{H}, \mathrm{NCHPh} 2)$, 3.56 (t, $2 \mathrm{H}, J=6.1 \mathrm{~Hz}, \mathrm{NCH}_{2}$ ), 3.48 (t, $\left.2 \mathrm{H}, \mathrm{J}=6.1 \mathrm{~Hz}, \mathrm{NCH}_{2}\right), 3.36(\mathrm{~m}, 2 \mathrm{H}$, $\mathrm{NHCH}_{2}$ ), 3.12 (t, $\left.2 \mathrm{H}, \mathrm{J}=5.7 \mathrm{~Hz}, \mathrm{NCH}_{2}\right), 1.96-1.89\left(\mathrm{~m}, 2 \mathrm{H}, \mathrm{CH}_{2}\right), 1.45(\mathrm{~s}$ 9H, $\left.\mathrm{CH}_{3}\right)$ ppm. ${ }^{13} \mathrm{C}$ NMR $\left(75 \mathrm{MHz}, \mathrm{CD}_{3} \mathrm{OD}\right): \delta=159.0(\mathrm{C}=\mathrm{O}), 155.8$ $(\mathrm{C}=\mathrm{NH}), 138.4\left(\mathrm{C}_{\mathrm{aAr}}\right), 130.2,130.0,129.7\left(\mathrm{CH}_{\mathrm{Ar}}\right), 80.7(\mathrm{CO}), 66.6$ $\left(\mathrm{NCHPh}_{2}\right), 52.2,49.2,44.4\left(\mathrm{NCH}_{2}\right), 38.9\left(\mathrm{NHCH}_{2}\right), 28.9\left(\mathrm{CH}_{3}\right), 22.4$ $\left(\mathrm{CH}_{2}\right)$ ppm. IR: vmax $=3321,3156,2962,1708,1637,1593,1542,1511$, $1361,1333,1242,1167,1132,1089,856,749,698 \mathrm{~cm}^{-1}$. HRMS (TOF MSES positive mode) $\mathrm{m} / \mathrm{z}$ calcd. for $\mathrm{C}_{24} \mathrm{H}_{33} \mathrm{~N}_{4} \mathrm{O}_{2}[\mathrm{M}]^{+}: 409.2604$; found 409.2586

(1R,2R)-[2-(3-Benzhydryl-3,4,5,6-tetrahydropyrimidin-2(1H)ylidenamino)-cyclohexyl] carbamic acid tert-butyl ester hydrobromide 12

White solid (88\%, conditions A); $\quad \mathrm{Mp}: 223-225^{\circ} \mathrm{C} ; \quad \mathrm{R}_{\mathrm{f}}: \quad 0.71$ (EtOH/DCM/Acetone 20/10/10); ${ }^{1} \mathrm{H}$ NMR $\left(300 \mathrm{MHz}, \mathrm{CD}_{3} \mathrm{OD}\right): \delta=7.47-$ $7.38(\mathrm{~m}, 6 \mathrm{H}, \mathrm{ArH}), 7.25-7.21(\mathrm{~m}, 4 \mathrm{H}, \mathrm{ArH}), 6.57$ (s, 1H, NCHPh 2$), 3.81-$ $3.64(\mathrm{~m}, 2 \mathrm{H}, \mathrm{NHCH}, \mathrm{NCH}), 3.57-3.34\left(\mathrm{~m}, 2 \mathrm{H}, \mathrm{NCH}_{2}\right), 3.16-3.03(\mathrm{~m}, 2 \mathrm{H}$, $\left.\mathrm{NCH}_{2}\right), 1.98-1.72\left(\mathrm{~m}, 7 \mathrm{H}, \mathrm{CH}_{2}\right), 1.51-1.32\left(\mathrm{~m}, 12 \mathrm{H}, \mathrm{CH}_{3}, \mathrm{CH}_{2}\right)$ ppm. ${ }^{13} \mathrm{C}$ NMR $\left(75 \mathrm{MHz}, \mathrm{CD}_{3} \mathrm{OD}\right): \delta=157.9(\mathrm{C}=\mathrm{O}), 155.6(\mathrm{C}=\mathrm{NH}), 138.4\left(\mathrm{C}_{\mathrm{qAr}}\right)$, 130.3, 130.2, 129.7 $\left(\mathrm{CH}_{\mathrm{Ar}}\right), 80.6(\mathrm{CO}), 67.0\left(\mathrm{NCHPh}_{2}\right), 63.6(\mathrm{NCH}), 51.4$ $(\mathrm{NHCH}), 44.6,41.3\left(\mathrm{NCH}_{2}\right), 34.0,30.6\left(\mathrm{CH}_{2}\right), 29.0\left(\mathrm{CH}_{3}\right), 26.1,25.9$, $22.9\left(\mathrm{CH}_{2}\right)$ ppm. IR: vmax $=3305,3191,2927,2864,2205,1684,1645$, 1562, 1511, 1444, 1317, 1156, 1077, 1018, 730, $698 \mathrm{~cm}^{-1}$. HRMS (TOF 
MSES positive mode) $\mathrm{m} / z$ calcd. for $\mathrm{C}_{28} \mathrm{H}_{39} \mathrm{~N}_{4} \mathrm{O}_{2}[\mathrm{MH}]^{+}:$: 463.3073; found : 463.3063. $[\alpha]_{578}^{20}:+28(c 0.5, \mathrm{MeOH})$.

\section{1,3-Bis(3-benzhydryl-3,4,5,6-tetrahydropyrimidin-2(1H)- ylideneamino)propane dihydrobromide 13}

White solid (87\%, conditions A); Mp: $159^{\circ} \mathrm{C}$; Rf: 0.10 (EtOAc/ $\mathrm{MeOH} / 30 \%$ aq. $\left.\mathrm{NH}_{3}: 48 / 50 / 2\right) ;{ }^{1} \mathrm{H}$ NMR $\left(300 \mathrm{MHz}, \mathrm{CD}_{3} \mathrm{OD}\right): \delta=7.51-7.26(\mathrm{~m}, 20 \mathrm{H}$, $\mathrm{ArH}), 6.60\left(\mathrm{~s}, 2 \mathrm{H}, \mathrm{NCHPh}\right.$ ), 3.64-3.42 (m, 8H, CH $\left.\mathrm{C}_{2} \mathrm{~N}\right), 3.21-3.15$ (appt t, $4 \mathrm{H}, J=5.7 \mathrm{~Hz}, \mathrm{CH}_{2} \mathrm{NCHPh}_{2}$ ), 2.19-2.04 (m, 2H, CH quint., $\left.4 \mathrm{H}, J=5.4 \mathrm{~Hz}, \mathrm{CH}_{2}\right)$ ppm. ${ }^{13} \mathrm{C}$ NMR $\left(75 \mathrm{MHz}, \mathrm{CD}_{3} \mathrm{OD}\right): \delta=156.1$ $(\mathrm{C}=\mathrm{NH}), 139.1\left(\mathrm{C}_{\mathrm{aAr}}\right), 130.9,130.7,130.5\left(\mathrm{CH}_{\mathrm{Ar}}\right), 67.5\left(\mathrm{NCHPh}_{2}\right), 50.3$, 48.9, 45.2 $\left(\mathrm{NCH}_{2}\right), 26.2,23.2\left(\mathrm{CH}_{2}\right)$ ppm. IR : vmax $=3306,3148,2935$, 2880, 1641, 1578, 1535, 1448, 1322, 1077, 726, $698 \mathrm{~cm}^{-1}$. HRMS (TOF MSES positive mode) $\mathrm{m} / \mathrm{z}$ calcd for $\mathrm{C}_{37} \mathrm{H}_{44} \mathrm{~N}_{6}[\mathrm{M}]^{2+}: 286.1813$; not found.

\section{$(R, R)$-1,2-Bis(3-benzhydryl-3,4,5,6-tetrahydropyrimidin-2(1H)-} ylideneamino)cyclohexane dihydrobromide 14

Light brown solid $\left(67 \%\right.$, conditions A); $\mathrm{Mp}: 290^{\circ} \mathrm{C}$; Rf: 0.15 (EtOAc/MeOH $/ 40 \%$ aq. $\left.\mathrm{NH}_{3}: 40 / 58 / 2\right) ;{ }^{1} \mathrm{H}$ NMR $\left(300 \mathrm{MHz}, \mathrm{CD}_{3} \mathrm{OD}\right): \delta=$ 7.52-7.23 (m, 20H, ArH), $6.63(\mathrm{~s}, 2 \mathrm{H}, \mathrm{NCHPh}), 4.33-4.30(\mathrm{bm}, 2 \mathrm{H}, \mathrm{CHN})$ 3.59-3.50 (m, 2H, CHHN), 3.32-3.14 (m, 4H, CHHN), 3.01-2.92 (m, 2H, $\mathrm{CH} H \mathrm{~N}), 2.27-1.60\left(\mathrm{~m}, 12 \mathrm{H}, \mathrm{CH}_{2}\right) \mathrm{ppm} .{ }^{13} \mathrm{C} \mathrm{NMR}\left(75 \mathrm{MHz}, \mathrm{CD}_{3} \mathrm{OD}\right): \delta=$ $156.4(\mathrm{C}=\mathrm{NH}), 139.1,138.5\left(\mathrm{C}_{\mathrm{qAr}}\right), 131.1,131.0,130.8,130.7,130.6$ $\left(\mathrm{CH}_{\mathrm{Ar}}\right), 67.9\left(\mathrm{NCHPh}_{2}\right), 59.6(\mathrm{NCH}), 46.1,42.2\left(\mathrm{NCH}_{2}\right), 30.9,25.9,23.4$ $\left(\mathrm{CH}_{2}\right)$ ppm. IR : vmax $=3440,3305,3191,3061,2924,2880,2860,1633$ 1554, 1526, 1452, 1322, 1073,746, $698 \mathrm{~cm}^{-1}$. HRMS (TOF MSES positive mode) $\mathrm{m} / \mathrm{z}$ calcd for $\mathrm{C}_{40} \mathrm{H}_{47} \mathrm{~N}_{6}[\mathrm{M}]^{+}: 611.3862$; found : 611.3851 . $[\alpha]_{578}^{20}:+62(c 1.9, \mathrm{MeOH})$. A crystal suitable for X-ray analysis was obtained by recrystallisation from isopropanol (see Supporting Information).

\section{8-Benzhydryl-3-phenyl-5,6,7,8-tetrahydro-imidazo[1,2,a]pyrimidin- \\ 2(4H)-one 16 \\ Conditions A. After two hours at $120^{\circ} \mathrm{C}$ under microwave irradiation, the} solution was concentrated under reduced pressure, giving one major product after trituration with small portions of diethyl ether. This residue was treated with aqueous $\mathrm{K}_{2} \mathrm{CO}_{3}$, extracted with EtOAc and then purified by flash chromatography (EtOAc/MeOH : $100 / 0$ to $90 / 10$ ) to afford 16 as a viscous clear oil (23\%); Rf: 0.50 (EtOAc); ${ }^{1} \mathrm{H} \mathrm{NMR}\left(300 \mathrm{MHz}, \mathrm{CDCl}_{3}\right): \delta$ $=7.41-7.19(\mathrm{~m}, 16 \mathrm{H}, \mathrm{ArH}, \mathrm{NCHPh}), 4.79(\mathrm{~s}, 1 \mathrm{H}, \mathrm{NCHPh}), 3.25-3.08(\mathrm{~m}$, $4 \mathrm{H}, \mathrm{NCH}_{2}$ ), 2.05 (appt quint., $\left.2 \mathrm{H}, \mathrm{J}=5.7 \mathrm{~Hz}, \mathrm{CH}_{2}\right)$ ppm. ${ }^{13} \mathrm{C} \mathrm{NMR}(75 \mathrm{MHz}$ $\left.\mathrm{CDCl}_{3}\right): \delta=184.9(\mathrm{CO}), 167.9(\mathrm{C}=\mathrm{NH}), 137.7,137.5,134.2\left(\mathrm{C}_{\mathrm{qAr}}\right), 128.9$, 128.7, 128.5, 128.2, 128.1, 127.8, 127.6, $127.1\left(\mathrm{CH}_{\mathrm{Ar}}\right), 69.7(\mathrm{CHPh})$, $64.0\left(\mathrm{CHPh}_{2}\right), 40.6,39.6,21.1\left(\mathrm{CH}_{2}\right) \mathrm{ppm}$. IR: vmax $=3089,3061,3026$ 2967, 2939, 2872, 2237, 1696, 1570, 1499, 1460, 1381, 1318, 1278, 908 722, $706 \mathrm{~cm}-1$. HRMS (TOF MSES positive mode) $\mathrm{m} / \mathrm{z}$ calcd for $\mathrm{C}_{25} \mathrm{H}_{24} \mathrm{~N}_{3} \mathrm{O}[\mathrm{MH}]^{+}: 382.1919$; found : 382.1916 .

\section{4-Benzhydryl-1-phenyl-[1,2,4]-triazepan-3-ylideneamine hydrobromide 17 \\ Beige solid (64\%, conditions B); Mp: $106^{\circ} \mathrm{C}$; Rf: 0.30 (EtOAc/MeOH $/ 40 \%$ aq. $\left.\mathrm{NH}_{3}: 93 / 5 / 2\right) ;{ }^{1} \mathrm{H} \mathrm{NMR}\left(300 \mathrm{MHz}, \mathrm{CD}_{3} \mathrm{OD}\right.$, major conformer $\left.66 \%\right): \delta$ $=7.55-6.90(\mathrm{~m}, 15 \mathrm{H}, \mathrm{Ar}), 6.36(\mathrm{~s}, 1 \mathrm{H}, \mathrm{NCHPh}), 3.76-3.58\left(\mathrm{~m}, 2 \mathrm{H}, \mathrm{NCH}_{2}\right)$, 3.45 (bs, $\left.2 \mathrm{H}, \mathrm{NCH}_{2}\right), 1.93$ (bs, $\left.2 \mathrm{H}, \mathrm{CH}_{2}\right)$ ppm. ${ }^{1} \mathrm{H}$ NMR $(300 \mathrm{MHz}$, $\mathrm{CD}_{3} \mathrm{OD}$, minor conformer $\left.33 \%\right): \delta=7.55-6.90(\mathrm{~m}, 15 \mathrm{H}, \mathrm{Ar}), 6.71(\mathrm{~s}, 1 \mathrm{H}$, $\mathrm{NCHPh}$ ), 3.76-3.58 (m, 2H, NCH$)_{2}$, 3.26 (appt t, $2 \mathrm{H}, \mathrm{J}=5.4 \mathrm{~Hz}, \mathrm{NCH}_{2}$ ), 2.14 (appt quint, $\left.2 \mathrm{H}, \mathrm{J}=5.4 \mathrm{~Hz}, \mathrm{CH}_{2}\right) \mathrm{ppm} .{ }^{3} \mathrm{C} \mathrm{NMR}\left(75 \mathrm{MHz}, \mathrm{CD}_{3} \mathrm{OD}\right.$, major and minor conformers): $\delta=159.0,150.7(\mathrm{C}=\mathrm{NH}), 146.1,139.0$, $138.9\left(\mathrm{C}_{\mathrm{Ar}}\right), 131.5,131.0,130.8,130.6,130.5,130.4,124.1,122.6$ 116.1, 115.6 $\left(\mathrm{CH}_{\mathrm{Ar}}\right), 69.4,67.7\left(\mathrm{CHPh}_{2}\right), 47.8,45.0\left(\mathrm{NCH}_{2}\right), 22.9\left(\mathrm{CH}_{2}\right)$ ppm. IR: vmax $=3239,3077,2963,2868,2300,1633,1592,1491,1444$, 1322, 738, $691 \mathrm{~cm}-1$. HRMS (TOF MSES positive mode) $\mathrm{m} / \mathrm{z}$ calcd for $\mathrm{C}_{23} \mathrm{H}_{25} \mathrm{~N}_{4}[\mathrm{M}]^{+}:$: 357.2079; found: 357.2073 . A crystal suitable for $\mathrm{X}$-ray analysis was obtained by recrystallisation from EtOAc (see Supporting Information).}

(R)-3-Benzyl-4-benzyloxymethyl-1-(1-phenylethyl)-3,4,5,6tetrahydropyrimidin-2(1H)-ylideneamine hydrobromide 21
Beige solid (90\%, conditions A); $\mathrm{R}_{\mathrm{f}}$ : $0.39\left(\mathrm{DCM} / \mathrm{MeOH} / \mathrm{aq} .30 \% \mathrm{NH}_{4} \mathrm{OH}\right.$ 95/5/1); ${ }^{1} \mathrm{H}$ NMR (300 MHz, $\mathrm{CD}_{3} \mathrm{OD}, 1: 1$ diastereoisomeric mixture): $\delta=$ 7.43-7.27 (m, 15H, ArH), 5.37-5.28 (m, 1H, NCH( $\left.\left.\mathrm{CH}_{3}\right) \mathrm{Ph}\right)$, 4.98-4.93 (m, $1 \mathrm{H}, \mathrm{NCH} H \mathrm{Ph}), 4.71-4.64(\mathrm{~m}, 1 \mathrm{H}, \mathrm{NCH} H \mathrm{Ph}), 4.52-4.40\left(\mathrm{~m}, 2 \mathrm{H}, \mathrm{OCH}_{2} \mathrm{Ph}\right)$, 3.72-3.53 (m, 3H, NCH, $\mathrm{CHCH}_{2} \mathrm{O}$ ), 3.42-3.33, 3.19-3.15, 3.01-2.87 (3m, $\left.2 \mathrm{H}, \mathrm{NCH}_{2}\right), 2.11-2.08,1.97-1.83\left(2 \mathrm{~m}, 2 \mathrm{H}, \mathrm{CH}_{2}\right), 1.65,1.56(2 \mathrm{~d}, 3 \mathrm{H}, \mathrm{J}=6.9$ $\left.\mathrm{Hz}, \mathrm{CH}_{3}\right)$ ppm. ${ }^{13} \mathrm{C}$ NMR $\left(75 \mathrm{MHz}, \mathrm{CD}_{3} \mathrm{OD}\right): \delta=156.4,156.3(\mathrm{C}=\mathrm{NH})$, 139.8, 139.4, 139.0, $136.6\left(\mathrm{C}_{\mathrm{qAr}}\right), 130.2,130.1,130.0,129.8,129.7$, $129.4,129.3,129.2,128.8,128.5,128.1,128.0,127.4\left(\mathrm{CH}_{\text {Ar }}\right), 74.6,74.5$ $\left(\mathrm{OCH}_{2} \mathrm{Ph}\right), 71.0,70.4\left(\mathrm{CHCH}_{2} \mathrm{O}\right), 57.5,57.5\left(\mathrm{NCH}\left(\mathrm{CH}_{3}\right) \mathrm{Ph}\right), 56.8,56.5$ $(\mathrm{NCH}), 54.0,54.0\left(\mathrm{NCH}_{2} \mathrm{Ph}\right), 39.3,39.3\left(\mathrm{NCH}_{2}\right), 26.2,25.8\left(\mathrm{CH}_{2}\right), 16.8$, $16.2\left(\mathrm{CH}_{3}\right)$ ppm. IR $:$ vmax $=3451,3309,3132,3057,2860,2201,1637$, 1566, 1538, 1491, 1451, 1321, 1073, 734, $694 \mathrm{~cm}^{-1}$. HRMS (TOF MSES positive mode) $\mathrm{m} / z$ calcd. for $\mathrm{C}_{27} \mathrm{H}_{32} \mathrm{~N}_{3} \mathrm{O}[\mathrm{M}]^{+}: 414.2545$; found : 414.2539.

\section{3-Benzyl-1-(4-methylbenzyl)-3,4,5,6-tetrahydropyrimidin-2(1H)-} ylidenamino-4-carbonitrile hydrobromide 22

Beige solid (91\%, conditions B); $\mathrm{Mp}: 81-83^{\circ} \mathrm{C}$; $\mathrm{R}_{\mathrm{f}}$ : 0.12 (DCM/MeOH/aq. $\left.30 \% \mathrm{NH}_{4} \mathrm{OH} 95 / 5 / 1\right) ;{ }^{1} \mathrm{H}$ NMR $\left(300 \mathrm{MHz}, \mathrm{CD}_{3} \mathrm{OD}\right): \delta=7.48-7.13(\mathrm{~m}, 9 \mathrm{H}$, ArH), 4.96-4.64 (m, 5H, NCH $\left.2 \mathrm{Ph}, \mathrm{NCH}_{2} \mathrm{PhCH}_{3}, \mathrm{CHCN}\right)$, 3.67-3.56 (m, $2 \mathrm{H}$, $\left.\mathrm{NCH}_{2}\right), 2.38-2.32\left(\mathrm{~m}, 5 \mathrm{H}, \mathrm{CH}_{3}, \mathrm{CH}_{2}\right) \mathrm{ppm} .{ }^{13} \mathrm{C} \mathrm{NMR}\left(75 \mathrm{MHz}, \mathrm{CD}_{3} \mathrm{OD}\right): \delta$ $=155.5(\mathrm{C}=\mathrm{NH}), 139.7,134.9,132.3\left(\mathrm{C}_{\mathrm{gAr}}\right), 131.0,130.4,130.2,128.5$, $128.2\left(\mathrm{CH}_{\mathrm{Ar}}\right), 117.7(\mathrm{CN}), 55.1,54.9\left(\mathrm{NCH}_{2} \mathrm{PhCH}_{3}, \mathrm{NCH}_{2} \mathrm{Ph}\right), 48.3(\mathrm{NCH})$, $45.0\left(\mathrm{NCH}_{2}\right), 26.0\left(\mathrm{CH}_{2}\right), 21.3\left(\mathrm{CH}_{3}\right) \mathrm{ppm}$. IR : vmax $=3306,3128,3022$, 2923, 2872, 2343, 1645, 1574, 1539, 1452, 1349, 805, 754, $702 \mathrm{~cm}-$ 1.cm ${ }^{-1}$. HRMS (TOF MSES positive mode) $\mathrm{m} / z$ calcd. for $\mathrm{C}_{20} \mathrm{H}_{23} \mathrm{~N}_{4}[\mathrm{M}]^{+}$: 319.1923; found : 319.1929 .

\section{3-Benzyl-4-methyl-1-(4-methylbenzyl)-3,4,5,6-tetrahydro-2(1H)-} pyrimidin-2(1H)-ylidenamino-4-carboxylic acid tert-butyl ester hydrobromide 23

White solid (25\%, conditions A); $\mathrm{Mp}$ : $97^{\circ} \mathrm{C}$; Rf: $0.25\left(\mathrm{CH}_{2} \mathrm{Cl}_{2} / \mathrm{MeOH}\right.$ : 90/10); ${ }^{1} \mathrm{H}$ NMR (300 MHz, $\mathrm{CD}_{3} \mathrm{OD}$ ): $\delta=7.45-7.18(\mathrm{~m}, 9 \mathrm{H}, \mathrm{ArH}), 4.91$ $4.53\left(\mathrm{~m}, 4 \mathrm{H}, \mathrm{NCH}_{2} \mathrm{Ph}, \mathrm{NCH}_{2} \mathrm{PhCH}_{3}\right)$, 3.55-3.35 (m, $\left.2 \mathrm{H}, \mathrm{NCH}_{2} \mathrm{CH}_{2}\right)$, 2.36$2.30\left(\mathrm{~m}, 2 \mathrm{H}, \mathrm{NCH}_{2} \mathrm{CH}_{2}\right), 2.36\left(\mathrm{~s}, 3 \mathrm{H}, \mathrm{CH}_{3} \mathrm{Ph}\right), 1.58\left(\mathrm{~s}, 3 \mathrm{H}, \mathrm{CCH}_{3}\right), 1.49(\mathrm{~s}$, $\left.9 \mathrm{H}, \mathrm{C}\left(\mathrm{CH}_{3}\right)_{3}\right)$ ppm. ${ }^{13} \mathrm{C} \mathrm{NMR}\left(75 \mathrm{MHz}, \mathrm{CD}_{3} \mathrm{OD}\right): \delta=173.1(\mathrm{C}=\mathrm{O}), 157.3$ $(\mathrm{C}=\mathrm{NH}), 140.2,138.0,133.3\left(\mathrm{C}_{\mathrm{qAr}}\right), 131.6,130.8,129.5,129.1,127.8$ $\left(\mathrm{CH}_{\mathrm{Ar}}\right), 85.7\left(\mathrm{COOC}\left(\mathrm{CH}_{3}\right)_{3}\right), 66.5\left(\mathrm{NC}_{9} \mathrm{CH}_{3}\right), 55.8,52.3\left(\mathrm{NCH}_{2} \mathrm{PhCH}_{3}\right.$, $\left.\mathrm{NCH}_{2} \mathrm{Ph}\right), 45.5\left(\mathrm{NCH}_{2} \mathrm{CH}_{2}\right), \quad 34.9\left(\mathrm{NCH}_{2} \mathrm{CH}_{2}\right), 28.9\left(\mathrm{C}_{\left.\left(\mathrm{CH}_{3}\right)_{3}\right), 24.6}\right.$ $\left(\mathrm{C}_{\mathrm{q}} \mathrm{CH}_{3}\right), 22.0\left(\mathrm{PhCH}_{3}\right)$ ppm. IR: vmax = 3308, 3126, 2975, 2927, 1727, 1636, 1588, 1536, 1449, 1374, 1353, 1148, 1127, 843, 750, $700 \mathrm{~cm}^{-1}$. HRMS (TOF MSES positive mode) $\mathrm{m} / z$ calcd for $\mathrm{C}_{25} \mathrm{H}_{34} \mathrm{~N}_{3} \mathrm{O}_{2}[\mathrm{M}]^{+}$: 408.2651; found : 408.2637 .

\section{Benzyl-(3-methyl-2-oxo-tetrahydrofuran-3-yl)cyanamide 24}

Colourless oil; Rf: 0.31 (PE/EtOAc : 60/40); ${ }^{1} \mathrm{H}$ NMR $\left(300 \mathrm{MHz}, \mathrm{CDCl}_{3}\right)$ : $\delta$ $=7.30-7.26(\mathrm{~m}, 5 \mathrm{H}, \mathrm{Ar}), 4.35-4.17\left(\mathrm{~m}, 2 \mathrm{H}, \mathrm{OCH}_{2}\right), \sim 4.26(\mathrm{~d}, \mathrm{~A}$ part of $\mathrm{AB}$ syst., $1 \mathrm{H}, J=14.4 \mathrm{~Hz}, \mathrm{NCH} H \mathrm{Ph}$ ), 4.16 (d, B part of $A B$ syst., $1 \mathrm{H}, J=13.8$ $\mathrm{Hz}, \mathrm{NCHHPh}), 2.69-2.60\left(\mathrm{~m}, 1 \mathrm{H}, \mathrm{OCH}_{2} \mathrm{CH} H\right), 2.27-2.18(\mathrm{~m}, 1 \mathrm{H}$, $\left.\mathrm{OCH}_{2} \mathrm{CHH}\right), 1.55\left(\mathrm{~s}, 3 \mathrm{H}, \mathrm{CH}_{3}\right) \mathrm{ppm} .{ }^{13} \mathrm{C} \mathrm{NMR}\left(75 \mathrm{MHz}, \mathrm{CDCl}_{3}\right): \delta=$ $174.2(\mathrm{C}=\mathrm{O}), 134.3\left(\mathrm{C}_{\mathrm{AAr}}\right), 128.8,128.7,128.6\left(\mathrm{CH}_{\mathrm{Ar}}\right), 114.3(\mathrm{CN}), 64.8$ $\left(\mathrm{OCH}_{2}\right), 61.6\left(\mathrm{NC}_{q} \mathrm{CH}_{3}\right), 51.5\left(\mathrm{NCH}_{2} \mathrm{Ph}\right), 34.7\left(\mathrm{OCH}_{2} \mathrm{CH}_{2}\right), 20.6\left(\mathrm{CqCH}_{3}\right)$ ppm. IR: vmax = 2991, 2916, 2887, 2212, 1776, 1723, 1497, 1453, 1385, $1219,1191,1099,1024,714 \mathrm{~cm}^{-1}$. HRMS (TOF MSES positive mode) $\mathrm{m} / \mathrm{z}$ calcd for $\mathrm{C}_{13} \mathrm{H}_{15} \mathrm{~N}_{2} \mathrm{O}_{2}[\mathrm{MH}]^{+}: 231.1135$; found : 231.1134 .

(1S,2R)-N-Benzyl-(1-cyano-2-phenylcyclopropyl)cyanamide 26 and $(1 R, 2 R)-N$-Benzyl-(1-cyano-2-phenylcyclopropyl)cyanamide 27: To a solution of the 3-bromopropyl cyanamide $25(64 \mathrm{mg}, 0.18 \mathrm{mmol})$.) in dry THF $(2.5 \mathrm{~mL})$ at $-78^{\circ} \mathrm{C}$ was added dropwise a $1.3 \mathrm{M}$ solution of LiHMDS in THF $(0.25 \mathrm{~mL})$ under argon. The reaction mixture was then allowed to stir, with reaction progress monitored by TLC. After completion, the reaction was quenched with a saturated aqueous $\mathrm{NH}_{4} \mathrm{Cl}$ solution and extracted with $\mathrm{CH}_{2} \mathrm{Cl}_{2}(3 \times 10 \mathrm{~mL})$. The combined organic extracts were 
dried over magnesium sulfate, filtered and evaporated under reduced pressure. The residue was purified by thin layer chromatography (EP/EtOAc 75/25).to give 26 as white needles (55\%) and 27 as a clear oil (31\%).

26: $\mathrm{Mp}: 120^{\circ} \mathrm{C}$; Rf: 0.41 (PE/EtOAc : 75/25); ${ }^{1} \mathrm{H}$ NMR $\left(300 \mathrm{MHz}, \mathrm{CDCl}_{3}\right.$ ): $\delta=7.47-7.39(\mathrm{~m}, 5 \mathrm{H}, \mathrm{ArH}), 7.36-7.31(\mathrm{~m}, 3 \mathrm{H}, \mathrm{ArH}), 7.04-7.01(\mathrm{~m}, 2 \mathrm{H}$ ArH), 4.48 (d, A part of AB syst., $1 \mathrm{H}, J=13.5 \mathrm{~Hz}, \mathrm{NCHH}), \sim 4.41$ (d, B part of $\mathrm{AB}$ syst., $1 \mathrm{H}, J=13.5 \mathrm{~Hz}, \mathrm{NCHH}), 2.77$ (appt. $t$, X part of $\mathrm{ABX}$ syst., $1 \mathrm{H}, \mathrm{CHPh}$ ), 1.98 (dd, A part of $\mathrm{ABX}$ syst., $1 \mathrm{H}, J=8.6 \mathrm{~Hz}, \mathrm{CHH}$ ), $\sim 1.86$ (dd, B part of $A B X$ syst., $1 \mathrm{H}, J=9.9 \mathrm{~Hz}, \mathrm{CH} H$ ) ppm. ${ }^{13} \mathrm{C}$ NMR (75 $\left.\mathrm{MHz}, \mathrm{CDCl}_{3}\right): \delta=132.7,132.1\left(\mathrm{C}_{\mathrm{qAr}}\right), 129.4,129.3,129.2,128.7,128.5$, $127.9\left(\mathrm{CH}_{\mathrm{Ar}}\right), 115.4(\mathrm{CN}), 113.0(\mathrm{NCN}), 55.7\left(\mathrm{CH}_{2} \mathrm{Ph}\right), 38.9(\mathrm{Cq}), 34.5$ $(\mathrm{CHPh}), 21.4\left(\mathrm{CH}_{2}\right)$ ppm. IR: vmax $=3101,3065,3033,2921,2215$, 1604, 1498, 1455, 1392, 1275, 1217, 1176, 1079, 1027, 776, 766, 745 $\mathrm{cm}^{-1}$. HRMS (TOF MSES positive mode) $\mathrm{m} / \mathrm{z}$ calcd for $\mathrm{C}_{18} \mathrm{H}_{16} \mathrm{~N}_{3}[\mathrm{MH}]^{+}$: 274.1344; found : 274.1338. [ $\alpha]_{578}^{20}:+80\left(c 2.7, \mathrm{CH}_{2} \mathrm{Cl}_{2}\right)$.

27: Rf: 0.50 (EP/EtOAc : 75/25); ${ }^{1} \mathrm{H}$ NMR $\left(300 \mathrm{MHz}, \mathrm{CDCl}_{3}\right): \delta=7.47$ 7.42 (m, 4H, ArH), 7.36-7.28 (m, 4H, ArH), 6.84-6.82 (m, 2H, ArH), 4.03 (d, A part of AB syst., $1 \mathrm{H}, J=12.3 \mathrm{~Hz}, \mathrm{NCH}), \sim 3.64$ (d, B part of AB syst. $1 \mathrm{H}, J=12.3 \mathrm{~Hz}, \mathrm{NCH} H$ ), 3.10 (appt. t, $\mathrm{X}$ part of $\mathrm{ABX}$ syst., $1 \mathrm{H}, \mathrm{CHPh}$ ), 2.16-2.02 (m, AB part of $\mathrm{ABX}$ syst., $\left.2 \mathrm{H}, \mathrm{CH}_{2}\right)$ ppm. ${ }^{13} \mathrm{C} \mathrm{NMR}(75 \mathrm{MHz}$, $\left.\mathrm{CDCl}_{3}\right): \delta=131.8,130.3\left(\mathrm{C}_{\mathrm{qAr}}\right), 129.4,129.3,129.2,128.9,128.7\left(\mathrm{CH}_{\mathrm{Ar}}\right)$, $117.8(\mathrm{CN}), 113.7(\mathrm{NCN}), 55.0\left(\mathrm{CH}_{2} \mathrm{Ph}\right), 36.3\left(\mathrm{C}_{\mathrm{q}}\right), 34.6(\mathrm{CHPh}), 19.4$ $\left(\mathrm{CH}_{2}\right)$ ppm. IR: vmax = 3092, 3066, 3032, 2924, 2219, 1604, 1498, 1455 1377, 1213, 1157, 1029, 778, 760, $744 \mathrm{~cm}^{-1}$. HRMS (TOF MSES positive mode) $\mathrm{m} / z$ calcd for $\mathrm{C}_{18} \mathrm{H}_{16} \mathrm{~N}_{3}[\mathrm{MH}]^{+}: 274.1344$; found : 274.1333. [ $\left.\alpha\right]_{578}^{20}$ : $+6\left(c 2.5, \mathrm{CH}_{2} \mathrm{Cl}_{2}\right)$.

\section{Acknowledgements}

The University of Versailles St-Quentin-en-Yvelines, University Paris-Saclay and the CNRS are acknowledged for funding.

Keywords: azetidines • von Braun reaction • guanidines

[1] W. R. J. D. Galloway, A. Isidro-Llobet, D. R. Spring, Nat. Commun. 2010, 1:80, doi: 10.1038/ncomms1081.

[2] For recent reviews on azetidines, including their preparations, see: a) Y. Dejaegher, N. M. Kuz'menok, A. M. Zvonok, N. De Kimpe, Chem. Rev. 2002, 102, 29-60. b) F. Couty, G. Evano, D. Prim, Mini Reviews in Organic Chemistry 2004, 1, 133-148 d) A. Brandi, S. Cicchi, F. M. Cordero, Chem. Rev. 2008, 108, 3988-4035. e) F. Couty, "Synthesis of Azetidines", In Science of Synthesis: Houben-Weyl Methods of Molecular Transformations; Enders, D., Ed.; Georg Thieme Verlag: New York, 2009; Vol. 40a, pp 773-817. f) F. Couty, G. Evano, B. Drouillat, O. David Eur. J. Org. Chem, 2013, 2045-2056. g) F. Couty, G. Evano, G. Synlett, 2009, 19, 3053-3064.

[3] J. T. Lowe, M. D. Lee IV, L. B. Akella, E. Davoine, E. J. Donckele, L. Durac, J. R. Duvall, B. Gerard, A. Holson, S. Joliton, S. Kesavan, B. C. Lemercier, H. Liu, J-C. Marié, C. A. Mulrooney, G. Muncipinto, M. Welzel-O'Shea, L. M. Panko, A. Rowley, B-C. Suh, M. Thomas, F. F. Wagner, J. Wei, M. A. Fowley, L. A. Marcaurelle, J. Org. Chem. 2012 77, 7187-7211

[4] M. Maetani, J. Zoller, B. Melillo, O. Vernho, N. Kato, J. Pu, E. Komer, S. L. Schreiber, J. Am. Chem. Soc. 2017, 139, 11300-11306. b) M Maetani, N. Kato, V. A. P. Jabor, F. A. Calil, M-C. Nonato, C. A. Sherer, S. L. Shreiber, ACS Med. Chem. Lett. 2017, 8, 438-442.

[5] K. Wright, B. Drouillat, J. Marrot, F. Couty, Eur. J. Org. Chem. 2017, 47, 2195-2201.

[6] J. v Braun, Chem. Ber. 1900, 33, 1438-1452. For a review, see: H. A. Hageman, 2011, Organic Reactions. 7:4:198-262.

[7] For reviews on $\mathrm{N}$-cyanamides, see: a) D. D. Nekrasov, Russ. J. Organ Chem. 2004, 40, 1387-1402. b) M-H Larraufie, G. Maestri, M. Malacria, C. Ollivier, L. Fensterbank, E. Lacôte, Synthesis, 2012, 44, 1279-1292. c) M. R. Rangha Prabhath, L. Williams, S. V. Baht, P. Sharma, Molecules, 2017, 22(4), 615 .

[8] R. C. Elderfield, H. A. Hageman, J. Org. Chem. 1949, 14, 605-637.

[9] For selected reviews, see: a) B. Maji, D. S. Stephenson, H. Mayr, ChemCatChem. 2012, 4, 2003-2009. b) J. E. Taylor, S. D. Bull, J. M. D. William, Chem. Soc. Rev. 2012, 41, 2109-2121. c) D. Leow, C-H. Tan Chem. Asian J. 2009, 4, 488-507. d) T. Ishikawa, T. Kumamoto, Synthesis, 2006, 5, 737-752. e) M. P. Cowles, Chem. Commun. 2009, 3659-3676.

[10] B. B. Snider, S. M. O'Hare, Tetrahedron Lett. 2001, 42, 2455-2458

[11] While we suppose that alkylation of the amine is the first step in cyclic guanidine formation, neither linear guanidines nor alkylated amine intermediates were observed or isolated from the reaction mixtures.

[12] Structures of compounds 14, 17, 26 have been deposited at the CCDC Cambridge database and have been allocated numbers 1858270 , 1858271, 1858272 respectively. For structure details, see supplementary material.

[13] Z. Zhu, A. W. Stamford, B. McKittrick, US2007/99898, 2007, A1.

[14] T. A. Nigst, J. Ammer, H. Mayr, Angew. Chem. Int. Ed. 2012, 51, 1353-1356. 


\section{FULL PAPER}

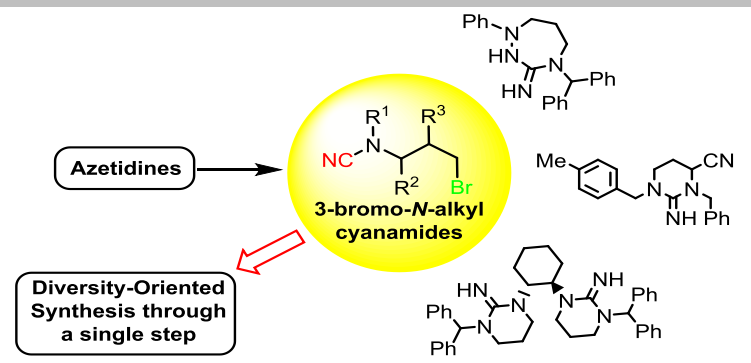

Guanidines, Cyanamides, Azetidines

Karen Wright*, Bruno Drouillat,

Laurence Menguy, Jérôme Marrot and François Couty

Page No. - Page No.

3-Bromo N-Alkyl Cyanamides as

Versatile Building Blocks

3-Bromo $\mathrm{N}$-alkyl cyanamides, accessible in one step from azetidines, were used as original building blocks for the synthesis of nitrogen heterocycles and functionalized cyclopropanes. 